\title{
Two Phase Flow Water Gas Separation in Biomass Energy Production
}

\author{
Prof. Yeong Ryu, State University of New York, Farmingdale
}

YEONG S. RYU graduated from Columbia University with a Ph.D. and Master of Philosophy in Mechanical Engineering in 1994. He has served as an associate professor of Mechanical Engineering Technology at Farmingdale State College (SUNY) since 2006. In addition, he has conducted various research projects at Xerox Corporation (1994-1995), Hyundai Motor Corporation (1995-1997), and New Jersey Institute of Technology (2001-2003). He has been teaching and conducting research in a broad range of areas of system identification and control of nonlinear mechatronic systems and vibrations in structures requiring precision pointing to eliminate the detrimental effects of such diverse disturbance sources. He has authored or co-authored more than 70 publications. His work currently focuses on the development and implementation of modeling and control of renewable energy systems, characterization of nanomaterials, photovoltaics, and nanoscale integrated systems. He is a member of the American Society of Mechanical Engineers (ASME), American Society for Engineering Education (ASEE) and the Materials Research Society (MRS).

\section{Dr. Hazem Tawfik, State University of New York, Farmingdale}

Prof. Tawfik obtained his Ph.D. in Mechanical Engineering, from University of Waterloo, Ontario, Canada. He has held a number of industrial \& academic positions and affiliations with organizations that included Brookhaven National Laboratory (BNL), Rensselaer Polytechnic Institute (RPI), Stony Brook University (SBU), Massachusetts Institute of Technology (MIT), Atomic Energy of Canada Inc., Ontario Hydro, NASA Kennedy, NASA Marshall Space Flight Centers, and the U.S. Naval Surface Warfare Center at Carderock, Md. Dr. Tawfik is the co-author of more than 60 research papers in the areas of Hydrogen Fuel Cells, Biomass Energy, Thermo- fluids and Two Phase Flow published in prestigious peer reviewed journals and conference symposiums. He holds numerous research awards and owns the rights to four patents in the Polymer Electrolyte Membrane (PEM) fuel cells area. Currently, Dr. Tawfik is a SUNY Distinguished Service Professor and the Director of the Institute for Research and Technology Transfer (IRTT) at Farmingdale State College of the State University of New York.

\section{Mr. Brandon Scott Weisberg, Farmingdale State College}

Junior Mechanical Engineering Technology Student at Farmingdale State College

\section{Dr. Gonca Altuger-Genc, State University of New York, Farmingdale}

Dr. Gonca Altuger-Genc is an Assistant Professor at State University of New York - Farmingdale State College in the Mechanical Engineering Technology Department. She is serving as the K-12 STEM Outreach Research and Training Coordinator at Renewable Energy and Sustainability Center at Farmingdale State College. Her research interests are engineering education, self-directed lifelong learning, virtual laboratories, and decision-making framework development for design and manufacturing environments. 


\title{
Two Phase Flow Water Gas Separation in Biomass Energy Production
}

\begin{abstract}
:
Recent concerns over the security and reliability of the world's energy supply has caused a flux in the research and development of renewable sources. A leading renewable source has been found in the biomass gasification of biological materials derived from organic matters such as wood chips, forest debris, and farm waste that are found in abundance in the USA. There is a very strong interest worldwide in the development of technologies that allow the coupling of biomass gasification and fuel cell systems to produce high-energy efficiency, clean environmental performance and near-zero greenhouse gas emissions. Biomass gasification is a process which produces synthesis gas (syngas) that contains $19 \%$ hydrogen and $20 \%$ carbon monoxide from organic matter.
\end{abstract}

In order to efficiently produce hydrogen from biomass that is capable of energizing the Proton Exchange Membrane (PEM) fuel cell to generate combined heat and power, ultraclean hydrogen with carbon monoxide $(\mathrm{CO})$ content less than 10 parts per million (ppm) must be fed to the PEM fuel cell to prevent the poisoning of its platinum catalyst.

Therefore, the separation of gas from water was found to be a very necessary process for water conservation. The considerable centrifugal force difference between gases and liquids like water is the essence of this research work for the two phase separation. A prototype for the two phase separator has been designed, fabricated and tested.

A major objective of this project is to enhance the educational experiences for engineering students while working on a team project resembling a realistic work environment similar to that of an industrial setting. The outcome of such learning experiences from this effort will be the design, implementation, theoretical analysis, model development and experimental application in the near future of an interdisciplinary project- oriented course for engineering students. This course will involve concepts from fluid mechanics, heat transfer, instrumentation, and data acquisition/analysis.

\section{Introduction:}

In the ever fluctuating global atmosphere and oil prices, fossil energy supply is possibly the most prominent issue to have an influence on our environment. An increasingly turbulent climate that reigns over this earth has interests vested deep in the market of energy supply. The real issue lies within the non-renewable sources of energy that reign today. The United States gets about 84\% of its total energy supply through the consumption of Oil, Coal, and Natural Gas, all of which are fossil fuels and are non-renewable. ${ }^{(1)}$ Biomass energy production is becoming an increasingly prominent alternative energy. Advancements in Biomass gasification would not only push further boundaries in research and engineering, but it would also help to stabilize the inherently unstable energy market that exists today.

Biomass energy is a process wherein organic matter is processed to yield an array of byproducts. The desirable synthesis gas, as previously stated, consists of $19 \%$ hydrogen and $20 \%$ carbon monoxide. Recent research and work aimed to further the development and optimization 
of the biomass process to increase extraction of hydrogen from the synthetic gas produced from the biomass process. In this process, for example, water and gases must be separated from their two phase flow to conserve water and allow the recycling of the water back to the steam generator. In order to perform two phase gas and water separation, one must first contextualize the separation with surrounding processes and phases.

Refer to Figure A to help put the separation process into context with the rest of Biomass gasification. The steps highlighted in yellow in the corresponding flow chart point out the portion of process that the relevant research went into.

\section{Procedure}

Part 1- Initial procedure of the separation of two phase flow is to create the two phases so that they can be separated by physical means. As the two phase flow leaves the module, as depicted in Figure B, the gas is in a mixture with a considerable portion of water vapor. The steam-syngas mixture must be separated later in the process but can only be done so in the form of a two phase flow. The synthesis gas would remain a gas and the steam would need to be changed into liquid water. This change is necessary because the method of separation depends upon relative densities.

The two phase flow is created by condensing the water vapor into liquid water. This condensation is necessary as the temperature of the synthesis gas/ steam mixture exiting the Steam Generator module is over 100 degrees Celcius. The condensation of the mixture is accomplished through the use of an automotive radiator and an electric fan. The choice of radiator was made with regards to final budget and economic viability. The electric fan is positioned in front of the radiator to draw fresh air into the radiator cooling fins. The mixture of syngas and water vapor travels through the high temperature hose from the steam generator into the radiator wherein a significant amount of heat is extracted due to increased surface area and heat exchange with the surrounding air. As the heat is removed, ideally all of the water vapor will be condensed into liquid water for further separation. After condensation, the two phase flow of gas and liquid water will be flowing together. This is wherein separation is required. 


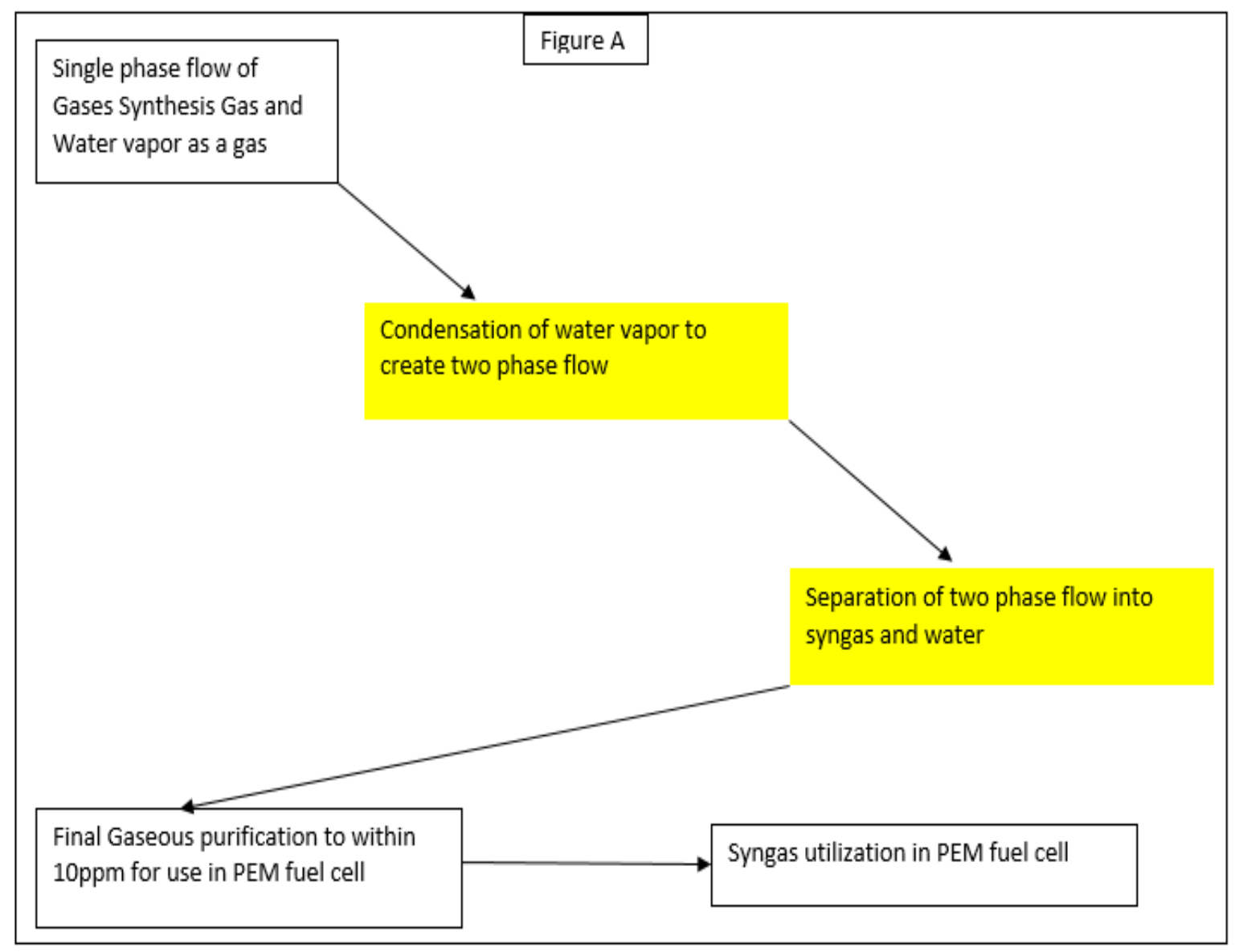

Figure (A) Process Schematic Diagram

Part 2- Following the establishment of a two phase flow of syngas and liquid water, the primary objective is now to separate the liquid water and syngas and route them effectively. The separation mechanism is based upon relative densities of the substances. In this instance, hydrogen gas is to be separated from liquid water. Since liquid water is usually $1 \mathrm{~g} / \mathrm{mL}$ and hydrogen gas is approximately $.000089 \mathrm{~g} / \mathrm{mL}^{(2)}$ the differential in density is significant enough to implement separation by density alone. 


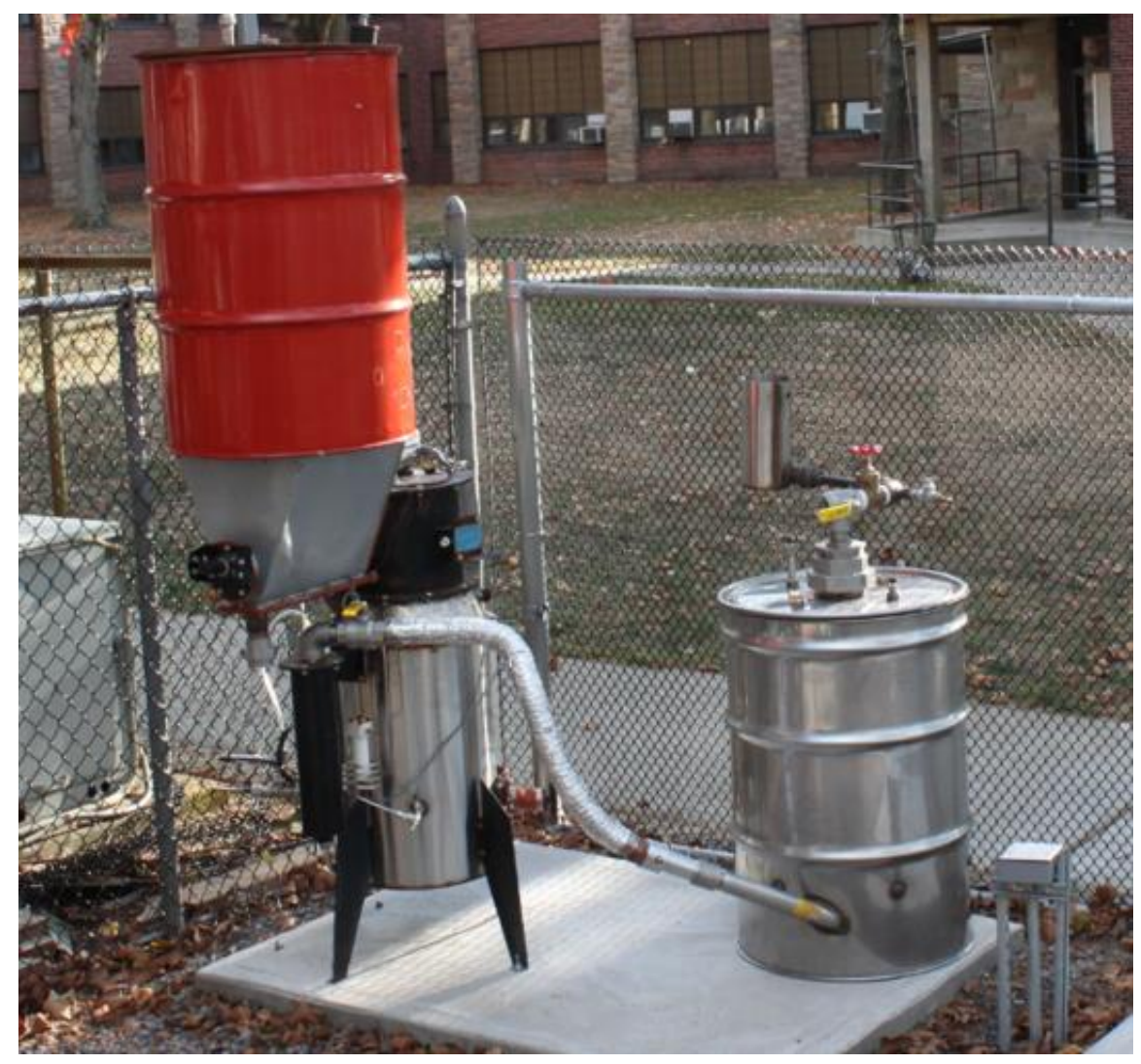

Figure (B) Two Phase Flow out of the Scrubbing Module shown in this figure

The method by which to separate the two phase flow by density is to utilize the relative weight of the liquid water to push it down in the flow. This relocation of liquid water towards the bottom of the synthesis gas mixture means that it is possible to put a drain at the bottom of the flow to extract the liquid water. The drain would be in the shape of a, "T." This, "T," shape is commonplace amongst plumbing hardware and will easily fit into the final budget with fairly minimal labor cost and time. This, "T," design can be seen in Figure $\mathrm{C}$ as well as the drainage tank. The idea being that as the denser liquid water sorts towards the bottom of the separator, the water would be extracted from a drain hole in the bottom of the coupling.

Now it was essential to be able to quantify the result in order to test the effectiveness of the methods of separation. It is not economically viable to implement sensors directly after and before the two phase separation. Therefore, it was necessary to develop an alternate method of quantifying data in order to interpret results. The way utilized to measure separation effectiveness is through the measurement of water flow rates going into and out of the system. Since water was introduced in the system, the flow rate is a controlled variable. In a perfect system then, the flow rate being introduced at the condenser should be the same as the flow rate being drained at the separator tank. Any difference in flow rates from input to drainage would be quantified as separation inefficiency (SI) and is used to calculate how effective the system is at separating the water and would help analysis.

\section{Equipment}


The equipment used in the experiment can be broken down into two parts; primary equipment and analysis equipment.

Primary equipment consists of all the equipment that is physically part of the biomass gasification module. Examples of primary equipment include the steam generator, the Scrubbing system, Condensation radiator, and the two phase separator.

The analysis equipment is made up of the equipment used to quantify the data during live experiments that would later be used to interpret the performance of the system. Examples of analysis equipment are temperature probes, drainage tank, glass graduated cylinders, stopwatch, and a data sheet.

\section{Data}

The data taken from the experiment is in the form of an excel spreadsheet shown in Table 1. The data to be taken into data tables are the Volume of Flow and time before and after two phase separation. These variables are expressed in the table below (Table 1). Another important variable in the data analysis is the temperature before and after separation. This was calculated using published values of saturation temperatures with known pressure before and after separation

Initial Temperature $=164$ Celsius

Final Temperature $=50$ Celsius 


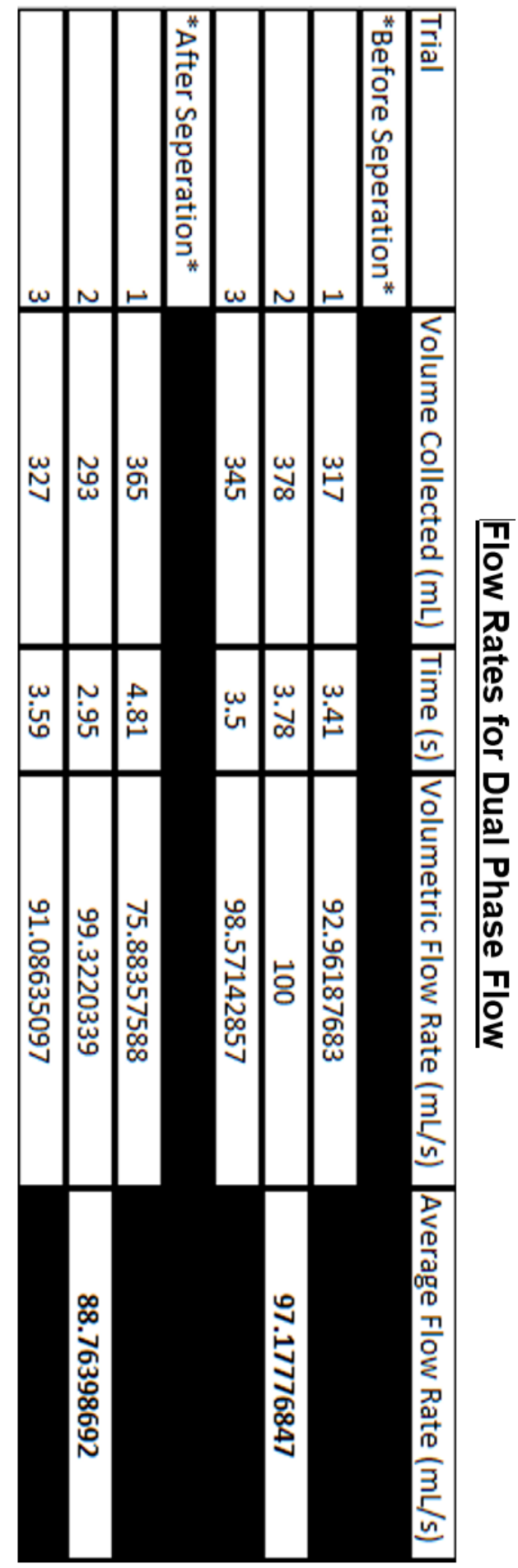

Table (1) Experimental Results

\section{Analysis}

Analysis and interpretation of the data taken is a necessary process to determine the effectiveness of the procedures taken in the experiment. The primary analysis of data to be done is to calculate 
the Separation Inefficiency (SI) Number, as discussed earlier. Since an average flow rate for before and after separation was collected, the SI number is calculated as follows.

$$
\begin{gathered}
S I=\left(\left(Q_{a}-Q_{b}\right) / Q_{b}\right) * 100 \\
S I=\frac{\frac{\left(\frac{88.8 m L}{S}-\frac{97.2 m L}{S}\right)}{97.2 m L}}{S} * 100 \\
S I=8.64 \% \text { Inefficient }
\end{gathered}
$$

*note- Qa $=$ Volumetric Flow Rate of drained water after separation

$\mathrm{Qb}=$ Volumetric Flow rate of water before separation

$\mathrm{SI}=$ Separation Inefficiency percentage number

This SI number of $8.64 \%$ inefficient is important to note as it can be converted into a separation efficiency percentage if just subtracted from $100 \%$

In effect, the separation efficiency of the gravity separator for Dual Phase Flow is $91.36 \%$ efficient.

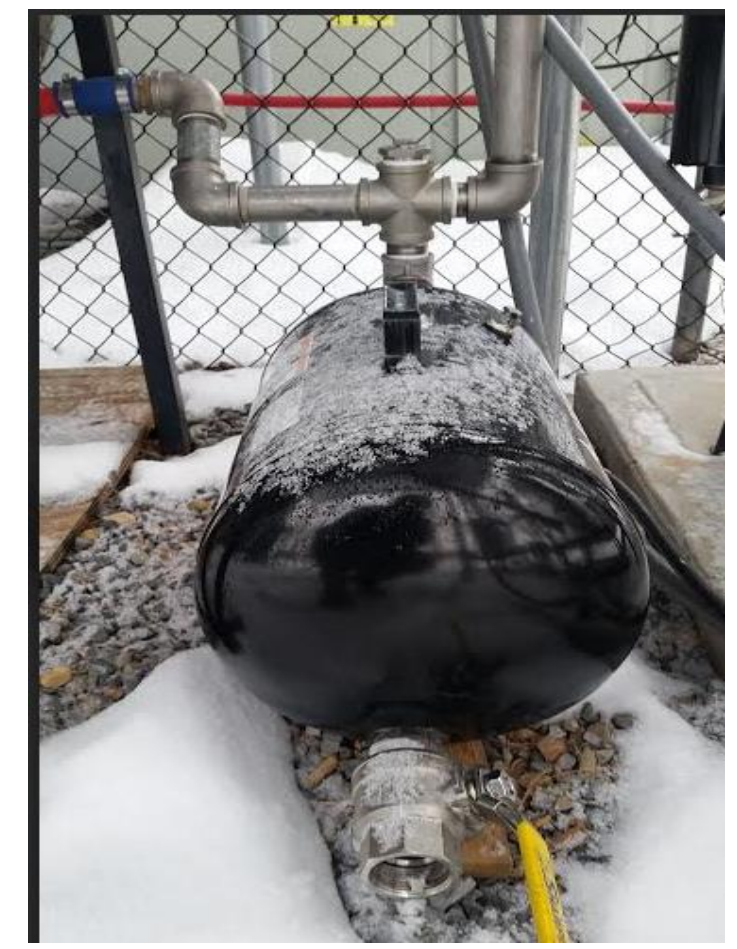

Figure (C) Separation system

\section{Impact in Engineering Technology Education}

Emerging technologies such as those involving alternate forms of energy are expected to play a major role in modern engineering technology curricula. The results presented in this paper 
involve expertise from multidisciplinary teams in our school of engineering technology; in particular, technology of biomass, control systems, fluid mechanics, thermodynamics, and software applications. Major parts of this work were performed as student projects by the first author who is a student in the school of engineering technology. Namely the student was involved in setting up the biomass system, developing code for control algorithm and data acquisition, and running the experiments at Farmingdale State College supported by the Department of Energy (DOE). It is expected that this lab setup will be used in future undergraduate senior projects for students in the departments of mechanical engineering technology. In addition, interdisciplinary courses in alternate forms of energy, biomass, solar energy systems, and control mechanisms could be developed in the future as outgrowth of these experimental setups and activities. Parts of the algorithms developed have also been used as examples in existing courses.

The performance of the biomass system is influenced by many different parameters. In this paper we analyzed the optimal performance of two phase flow water gas separation in biomass system. Temperature is an important parameter to the maximize power. We will continuously investigate the relationship between temperature, humidity, time and power. For real life applications, we need to develop a more sophisticated system to consider many parameters in the extended running of biomass system.

\section{Conclusion}

Through experimentation and analysis, the efficiency of the Gas-water separation has been determined. While this number of $91.36 \%$ sounds like a reasonable efficiency percentage, it is not sufficient to stop the two phase separation and refine the purification of synthesis gas at this point. It would be reasonable to enhance the two phase separation to at least $97 \%$ efficiency in future gas-water separation experiments with a few key refinements. The refinements can be made in two possible areas

The first set of refinements can occur within the condensation phase of the experiment wherein a single phase flow is divided into a dual phase flow. It has become apparent, from careful examination of end products, that not all of the input water vapor is being condensed into liquid water. This is causing the two phase flow to mix more homogenously and creates an inefficient separation mixture in terms of splitting relative densities. Possible augmentations to the system to increase condensation would be changes to the automotive radiator or the radiator fan system. It would be possible to put a bigger fan in place to draw more air over the cooling fins of the radiator. Another possible improvement would be to place a second automotive radiator connected in series with the first radiator. A second radiator would ensure complete vapor condensation and would help with splitting into a more defined two phase flow.

The second set of refinements comes in the place of the Two Phase separator. Based upon the incomplete gas-water separation, it is probable that the method of density separation can be augmented through the use of some basic improvements. The idea of centrifugal separation may be employed to increase separation efficiency. Centrifugal Separation is defined as the, "Process to separate isotopes in which heavy atoms are split from the lighter atoms by centrifugal forces. 
The separation factor depends on the mass difference of the isotopes to be separated." (3) It would be possible to implement a curved separator to force the two phase flow along a curved path and increase the density separation as a result.

\section{Bibliographic Information}

(1) "The National Academies." Fossil Fuels - Web. 15 Jan. 2015. <http://needtoknow.nas.edu/energy/energy-sources/fossil-fuels/>.

(2) "Density of Gases." Density of Gases. Web. 8 Jan. 2015. $<$ http://www.elmhurst.edu/ chm/vchembook/123Adensitygas.html>.

(3) "Gas Centrifuge Process." Gas Centrifuge Process. Web. 18 Jan. 2015. $<$ http://www.euronuclear.org/info/encyclopedia/g/gascentrifuge.htm>. 